TITLE:

\title{
Verification of validity of gait analysis systems during treadmill walking and running using human pose tracking algorithm
}

\section{AUTHOR(S):}

Ota, Megumi; Tateuchi, Hiroshige; Hashiguchi, Takaya; Ichihashi, Noriaki

\section{CITATION:}

Ota, Megumi ... [et al]. Verification of validity of gait analysis systems during treadmill walking and running using human pose tracking algorithm. Gait \& Posture 2021, 85: 290297

\section{ISSUE DATE:}

2021-03

URL:

http://hdl.handle.net/2433/261778

\section{RIGHT:}

(c) 2021. This manuscript version is made available under the CC-BY-NC-ND 4.0 license

http://creativecommons.org/licenses/by-nc-nd/4.0/.; The full-text file will be made open to the public on 1 March 2022 in accordance with publisher's 'Terms and Conditions for Self-Archiving'., この論文は出版社版でありません。引用の際 には出版社版をご確認ご利用ください。; This is not the published version. Please cite only the published version. 


\section{Title}

Verification of validity of gait analysis systems during treadmill walking and running using human pose tracking algorithm

\section{Short title}

Validity of human pose tracking algorithm

\section{Author names and affiliations}

Megumi Ota ${ }^{1)}$, Hiroshige Tateuchi ${ }^{1)}$, Takaya Hashiguchi ${ }^{2)}$, Noriaki Ichihashi ${ }^{3)}$

1. Department of Preventive Physical Therapy, Human Health Sciences, Graduate School of Medicine, Kyoto University, Kyoto, Japan

2. Mixi Incorporated, Tokyo, Japan

3. Department of Physical Therapy, Human Health Sciences, Graduate School of Medicine, Kyoto University, Kyoto, Japan

\section{Corresponding author}

Ota Megumi

Department of Preventive Physical Therapy, Human Health Sciences, Graduate School of Medicine, Kyoto University, Kyoto 606-8507, Japan

53 Kawahara-cho, Shogoin, Sakyo-ku, Kyoto, Japan

Tel/Fax: +81-75-751-3948

Email: ota.megumi.7e@kyoto-u.ac.jp

\section{Acknowledgments}

We thank all of our study subjects for their participation. This study was supported by a joint research fund from Smart Health Incorporated. We express our sincere thanks to Mr. Yasuhiro Ogino. 


\section{Abstract}

Background: The human tracking algorithm called OpenPose can detect joint points and measure segment and joint angles. However, the validity of gait analysis using OpenPose has not been examined yet.

Research question: What is the validity of OpenPose-based gait analysis?

Methods: Twenty-four healthy young people participated in this study. The participants were assessed during walking and running. Pelvic segment angles, and hip, knee, and ankle joint angles during treadmill walking and running were measured using VICON. Simultaneously, images were captured using digital cameras from the right and back sides. After processing with OpenPose, the corresponding angles were measured from the estimated joint points. To validate these estimations, linear regression analysis was performed, and intraclass correlation coefficients [ICCs $(2,1)]$ between the data obtained by OpenPose and VICON were calculated. Furthermore, the agreement between the data obtained by OpenPose and VICON was assessed by Bland-Altman analysis.

Results: For most ranges of motion (ROM) in the sagittal plane, the hip, knee, and ankle joints had large coefficients of determination, without proportional biases. For most peak angles in the sagittal plane, the knee and ankle joints had large coefficients of determination without proportional biases, although the hip joint had nonsignificant coefficients of determination and proportional biases. In particular, for the hip flexion-extension ROM and peak knee flexion angle during running and the knee ROM during slow walking, the ICCs showed good to excellent agreement. However, for the parameters of the pelvis and hip joint in the frontal plane, there were nonsignificant coefficients of determination and poor ICCs with fixed and proportional biases.

Significance: The lower limb ROM in the sagittal plane during gait can be measured by the OpenPose-based motion analysis system. The markerless systems have the advantage of being more economical and convenient than conventional methods.

(295 words / 300)

\section{Keywords}

Gait analysis; Human pose tracking algorithm; OpenPose 


\section{$1 \quad$ Introduction}

Experts conduct gait analyses to identify motion abnormalities in clinical evaluations. The gait parameters for quantitative evaluations include the step length, step width, gait velocity, steps, cadence, gait cycle time, step duration, joint angles, heart rate, and oxygen saturation. Simple quantitative evaluations such as a $10 \mathrm{~m}$ or less platform walk test for step length, gait velocity, steps [1,2], and a 6 min walking test [3] and incremental shuttle walking test for gait endurance [4] are used in clinical and research fields.

For the quantitative evaluation of joint motion during walking, gait analysis is performed by observation. However, it is difficult to confirm that the data are objective [5]. Therefore, for more detailed gait analysis, the marker-based system and accelerometer are used conventionally. Many kinds of studies using marker-based system devices such as VICON have been published $[6,7]$. However, conventional motion analysis systems such as the marker-based system and accelerometer have some disadvantages. Conventional motion analysis systems are expensive; moreover, time and technical skills are needed to attach makers [8] or sensors. Therefore, they are used in limited special environments such as laboratories. In most clinical fields, it is therefore difficult to establish an environment where motion evaluations could be performed using conventional motion analysis systems.

In recent years, video analysis technologies, especially markerless systems using a human pose tracking algorithm, have improved tremendously. Kinect, which was released in 2010, comprises RGB cameras equipped with an infraredbased depth sensor and can observe motion without requiring the attachment of reflective markers and the use controllers, making it possible to recognize the position and orientation of a part of the human body. The Kinect motion analysis system is easier to operate, more portable, and more economical than conventional motion analysis systems. However, previous studies reported significant differences between Kinect and conventional motion analysis systems such as VICON or Optotrak [9-14]. In a previous study [9] analyzing platform gait analysis using Kinect and VICON, the correlations indicating validity and intraclass correlation coefficients [ICCs] indicating reliability were determined. The correlations for most of spatiotemporal parameters were large. However, for the kinematic parameters such as the lower limb angles, the correlations were medium or small. Similarly, in another previous study [10] analyzing treadmill gait using Kinect and VICON, although the correlations for most of the spatiotemporal parameters and knee angles were medium or large, the 
correlations for the hip angles in the sagittal plane were small. Therefore, although Kinect can be used for spatiotemporal measurements, it may not be a suitable valid tool for obtaining kinematic measurements during walking.

OpenPose is a posture tracking algorithm employed to estimate the posture of multiple people from the images captured by a monocular camera using a convolutional neural network, which is a type of deep learning network $[15,16]$. Similar to Kinect, OpenPose does not require the attachment of makers or sensors to the body, and does not require technical expertise. Furthermore, unlike Kinect, OpenPose does not need special cameras. OpenPose is a real-time system for feature point detection on single images captured by a standard digital camera. Therefore, it is possible to record the motion of each participant using one digital camera and analyze the motions of the trunk and limbs from the captured images. In addition, OpenPose is an open source software that can be found in the OpenCV library [17-19], and its license allows for its noncommercial use for free. Moreover, its commercial use expenses are lower than those associated with conventional motion analysis devices, making it more convenient and economical than conventional portable devices, and it does not require a laboratory setting. If it is indeed possible to analyze gait using OpenPose, it could be more applicable in clinical fields. Our previous study has already demonstrated the reliability and validity of motion analysis during squat using OpenPose. Device test-retest reliability was in nearly complete agreement, and the angles of the trunk and lower limb joints during squat, measured using OpenPose, were associated with the corresponding angles measured using VICON [20]. In addition to our study [20], the joint positions during walking were estimated using OpenPose and conventional marker-based systems in recently published studies $[21,22]$ and the differences in corresponding joint positions were presented. However, studies on the validity of OpenPose for measuring the segment and joint angles during walking and more dynamic tasks, such as running, have not been conducted. Therefore, the purpose of this study was to clarify correlations and agreements of gait analysis using OpenPose with VICON during walking and running, and confirm the validity of gait analysis using OpenPose.

\section{Methods}

\subsection{Participants}

The participants were 24 healthy young people (17 males and 7 females; mean age: $26.1 \pm 4.6$ years; mean height: 
musculoskeletal injuries, neurological disorders, fractures, and those who had undergone surgery in the extremities and trunk were excluded. This study was approved in advance by the ethics committee of Kyoto University (approval number: R1823), and all the participants provided informed consent. GPower V.3.1.9.4 was used to determine the appropriate sample size for this study. For linear regression analyses, 20 participants needed to be enrolled to obtain an effect size exceeding 0.35 , which is deemed large [21], and achieve a power of $80 \%$ with a $5 \%$ error level in a one-tailed test. Previous studies targeted at healthy people on gait analysis using Kinect and VICON indicated that at least 20 participants were required [9-12]. Thus, we enrolled 24 participants in our study. OpenPose detects feature points from changes in pixels. To prevent misidentification, the participants were asked to wear appropriately sized light-colored garments that were not loose and did not interfere with motion.

\subsection{Motion task}

In each experimental trial, the participants were randomly asked to perform treadmill walking and running. The velocities were as follows: slow walking: $2.5 \mathrm{~km} / \mathrm{h}(0.69 \mathrm{~m} / \mathrm{s})$, moderate walking: $4.0 \mathrm{~km} / \mathrm{h}(1.11 \mathrm{~m} / \mathrm{s})$, fast walking: 5.5 $\mathrm{km} / \mathrm{h}(1.53 \mathrm{~m} / \mathrm{s})$, and running: $8.5 \mathrm{~km} / \mathrm{h}(2.36 \mathrm{~m} / \mathrm{s})$. After several practice trials, at least four gait cycles during which the participants remained stable were recorded.

\subsection{Data collection and processing}

For conventional measurement of the segment and joint angles, kinematic measurements by three-dimensional (3D) motion analysis systems were recorded using VICON (Vicon Nexus; Vicon Motion Systems Ltd., Oxford, England) with eight cameras. Kinematic measurements by two-dimensional (2D) analysis using human pose tracking algorithms were recorded using two digital cameras. The digital video cameras were set at $1.8 \mathrm{~m}$ at the back and to the right of the participants. The camera lens height was set to $91.5 \mathrm{~cm}$. The motion images were recorded from the right and back side. The feature points of each joint were estimated using OpenPose from the captured images of the participants during treadmill walking and running. The cut-off frequency of the filter was determined using residual analysis [23]. The data from both devices were recorded at a sampling rate of $60 \mathrm{~Hz}$ with a low-pass filter using a $6 \mathrm{~Hz}$ cut-off frequency.

\subsection{Data analysis}

The peak angles of pelvic elevation and depression, hip flexion, extension, abduction, adduction, knee flexion and extension, ankle dorsiflexion, and plantar flexion were measured, and the ranges of motion (ROM) during each 
gait cycle were calculated. Heel contact was identified from the images in each frame visually, and the gait cycle was defined. The peak angles and ROM were calculated, and the mean values of four successful gait cycles were used for the analyses.

According to the VICON Plug-in Gait marker placement protocol, 16 reflective markers were attached bilaterally to the anterior superior iliac spine (ASIS), posterior superior iliac spine (PSIS), lateral thigh, lateral femoral epicondyle, lateral shank, lateral malleolus, second metatarsal head, and calcaneus. In addition to these markers, the center of each joint was estimated from the following actual measured values: inter-anterior superior iliac spine distance, leg length (the ASIS to the lateral malleolus), knee width, and ankle width. First, the pelvis segment coordinate system was defined from the ASIS and PSIS markers. The pelvic origin was taken as the midpoint of both ASIS markers. The Y-axis was the direction from the right ASIS marker to the left ASIS marker. The X-axis was the direction starting from the midpoint of both PSIS markers to the midpoint of both ASIS markers and continuing forward. The $\mathrm{Z}$-axis was the direction perpendicular to the $\mathrm{X}$ and $\mathrm{Y}$ axes and continuing upward. The thigh was defined as a coordinate system as follows: The Z-axis was the direction from the center of the knee joint to the center of the hip joint. The Y-axis was taken parallel to the line from the center of the knee joint to the lateral femoral epicondyle marker. The $\mathrm{X}$-axis was the direction perpendicular to the $\mathrm{Y}$ and $\mathrm{Z}$ axes and continuing forward. The shank was defined as a coordinate system as follows: The Z-axis was the direction from the center of the ankle joint to the center of the knee joint. The Y-axis was taken parallel to the line from the center of the ankle joint to the lateral malleolus marker. The $\mathrm{X}$-axis was the direction perpendicular to the $\mathrm{Y}$ and $\mathrm{Z}$ axes and continuing forward. The foot was defined as a coordinate system as follows: The Z-axis was the direction from the second metatarsal head to calcaneus markers and continuing forward. The Y-axis was taken parallel to the line from the center of the ankle joint to the lateral malleolus marker. The $\mathrm{X}$-axis was the direction perpendicular to the $\mathrm{Y}$ and $\mathrm{Z}$ axes and continuing downward. The clinical manager software in VICON was used to calculate the pelvic angle on the global coordinate system, and the relative angles between the coordinate systems of each segment in the lower limb, using Euler angles. The angle of the pelvic elevation and depression was measured between the transverse axis in the frontal plane of the laboratory (the horizontal axis perpendicular to the participant's axis of progression) and the pelvic Y-axis. The Y-axis represented hip flexion and extension, knee flexion and extension, and ankle dorsiflexion and plantar flexion. The X- 
axis represented hip abduction and adduction.

With respect to OpenPose, the segment and joint angles were measured from the estimated feature points of each joint. Figure 1 presents the placement and definition of each feature point, and the segment and joint angles between each feature point were measured from the obtained marker coordinates as shown in Table 1.

\subsection{Statistical analysis}

First, to confirm that the results obtained by VICON can be predicted from the data obtained by OpenPose, linear regression analyses were performed by using the data obtained by OpenPose as the independent variables and the data obtained by VICON as the dependent variables. The correlation coefficients (r) were determined and values between 0.1 and 0.3 and those between 0.3 and 0.5 and those greater than 0.5 represented small, medium, and large correlations, respectively [24]. Therefore, the coefficients of determination $\left(\mathrm{R}^{2}\right)$ between 0.01 and 0.09 showed a small correlation, those between 0.09 and 0.25 showed a medium correlation, and those greater than 0.25 showed large correlations. Next, to confirm if the data obtained by OpenPose agreed with the data obtained by VICON, the ICCs ( 2 , 1) between the data obtained by OpenPose and VICON were calculated. The ICCs $<$ than 0.5 showed a poor agreement, those between 0.5 and 0.75 showed a moderate agreement, those between 0.75 and 0.9 showed a good agreement, and those greater than 0.90 showed an excellent agreement [25]. Furthermore, agreement between the data obtained by OpenPose and VICON was assessed by the Bland-Altman analysis. The level of significance was set at 0.05 . The statistical analyses were performed using IBM SPSS Statistics, version 26 (IBM Japan Ltd., Tokyo, Japan).

\section{Results}

Typical examples of temporal changes during moderate walking are indicated in Figure 2. The mean values \pm standard deviations for each peak angle and ROM using OpenPose and VICON are listed in Table 2.

For most parameters of the pelvis, the coefficients of determination were nonsignificant, and the ICCs showed poor agreement $(\mathrm{ICC}=-0.06-0.23)$. For the pelvis elevation angle during running and pelvis depression angle during fast walking only, there were medium coefficients of determination $\left(\mathrm{R}^{2}=0.18-0.20, \mathrm{p}<0.05\right)$ (Table 3$)$. There were fixed and proportional biases, and as the angles increased, the OpenPose values trended smaller than the VICON values (Table 4).

For the hip flexion-extension ROM, the coefficients of determinations were large $\left(\mathrm{R}^{2}=0.55-0.81, \mathrm{p}<0.01\right)($ Table 
3), and there were fixed biases but no proportional biases (Table 4). In particular, for the hip flexion-extension ROM during running, the ICC showed good agreement $(\mathrm{ICC}=0.86)($ Table 3$)$. However, for most parameters of the hip flexion and extension angles, the coefficients of determination were nonsignificant, and the ICCs were poor (ICC $=0.02-0.48)$ (Table 3). Moreover, there were fixed and proportional biases, and as the angles increased, the OpenPose values became smaller than the VICON values (Table 4). For most parameters of the hip abduction and adduction angles, although the coefficients of determinations were nonsignificant, or medium to large $\left(\mathrm{R}^{2}=0.21-0.53, \mathrm{p}<0.05\right)$, and the ICCs were poor $($ ICC $=0.03$ -0.56) (Table 3). Moreover, there were fixed and proportional biases, and as the angles increased, the OpenPose values became smaller than the VICON values (Table 4). For most parameters of the hip abduction-adduction ROM, the coefficients of determination were nonsignificant, and the ICCs were poor ( ICC $=0.03-0.11)$ (Table 3$)$. Additionally, there were fixed and proportional biases, and as the angles increased, the OpenPose values became smaller than the VICON values (Table 4).

For most of the knee angles and ROM, the coefficients of determinations were large $\left(\mathrm{R}^{2}=0.41-0.92, \mathrm{p}<0.01\right)$ (Table 3), and there were fixed biases but no proportional biases (Table 4). In particular, for the knee flexion angle during running and the knee ROM during slow walking, the ICCs showed excellent agreement (ICC $=0.91-0.93$ ).

For most of the ankle ROM, the coefficients of determinations were large $\left(\mathrm{R}^{2}=0.49-0.66, \mathrm{p}<0.01\right)$. Although the ICCs showed moderate correlations (ICC $=0.49-0.59)$, the $95 \%$ confidence intervals $[\mathrm{CI}]$ ranged widely. For most parameters of the ankle angles, the coefficients of determination were nonsignificant, or medium to large $\left(\mathrm{R}^{2}=0.21-0.52\right.$, $\mathrm{p}<0.05$ ), and the ICCs were poor to moderate (ICC $=0.11-0.68)$ (Table 3$)$. However, for most of the ankle angles and ROM, there were fixed biases but no proportional biases (Table 4).

\section{Discussion}

We observed large coefficients of determination without proportional biases for most parameters of the hip flexionextension ROM, and the knee and ankle joints. The ICCs showed good to excellent agreement, particularly for the hip flexion-extension ROM during running, peak knee flexion angle during running, and knee ROM during slow walking. However, for most parameters of the hip joint, except for the flexion-extension ROM, and the pelvis, the coefficients of determination were nonsignificant, and the ICCs were poor, with fixed and proportional biases. In our previous study, 
and validity of the approach were confirmed [20]. Moreover, in a previous study in which cadence was measured using

OpenPose, clinical application of the motion analysis using OpenPose was suggested [26]. In recent studies, the joint positions during walking were estimated using OpenPose and marker-based systems, and the differences in the corresponding joint positions, estimated from the two different measurement methods, were presented [21, 22]. However, the degree of agreement between the angles measured using OpenPose with the angles measured using VICON and the type of bias have not been confirmed yet, and the validity of OpenPose-based gait analysis has not been verified. This study is the first attempt to apply OpenPose to the measurement of segment and joint angles during walking and running.

First, there were large coefficients of determinations between the data obtained by OpenPose and VICON in the hip flexion-extension ROM and most parameters of the knee and ankle joints. Moreover, there were no proportional biases. For most parameters of the lower limb ROM in the sagittal plane, the ICCs were moderate to excellent; specifically for the hip flexion-extension ROM during running, knee flexion angle during running, and knee ROM during slow walking, the 95\% CIs were in a narrow range. Therefore, the validity of OpenPose was proved when measuring the lower limb ROM in the sagittal plane. Although the motions in the sagittal plane without transverse plane rotation of the knee and ankle joints obtained by OpenPose and VICON agreed, the motions in the frontal plane with transverse plane rotation of the pelvis and hip joints obtained by OpenPose and VICON not agreed. This is because, while VICON is a 3D motion analysis system, OpenPose provides only 2D motion data for images captured by one digital camera. Thus, motions with transverse plane rotation were not measured accurately. In particular, because the angles of the hip joint and pelvis in the frontal plane were small, the biases between the data obtained by OpenPose and VICON increased in motions with transverse plane rotation. However, for motion tasks without transverse plane rotation, the 2D analysis using OpenPose might provide valid results. Furthermore, there are differences in angle measurement methods between OpenPose and VICON, thereby measurement of the hip joint angles was affected by motion of pelvis and spine. In VICON, the hip angle in the sagittal plane was defined as the angle between the femur with respect to the pelvis using local coordinates, whereas in OpenPose, it was defined as the angle of the thigh with respect to the trunk using $2 \mathrm{D}$ coordinates. Therefore, the pelvis and spine positions affect the hip angles. 
determination were nonsignificant, and the ICCs were poor; moreover, fixed and proportional biases existed. However, for the hip flexion-extension ROM, the coefficients of determination were large, and there were no proportional biases. The motion and position of the pelvis or spine during walking or running varies depending on the participant. In walking or running with motions or varying positions of the pelvis or spine, differences between the hip angle data obtained by OpenPose and VICON might occur. For example, in walking or running with the forward pelvis tilt, the hip flexion angles measured by OpenPose are smaller than those measured by VICON. However, in walking or running while maintaining the pelvis tilt or spine flexion, although the hip flexion and extension angles obtained by OpenPose and VICON are different, the differences between the motion analyses systems in the hip flexion-extension ROM are not noticeable. The effects of the positions of the pelvis or spine could be eliminated in the hip flexion-extension ROM. For the hip flexion-extension ROM and most parameters of the knee and ankle joints, only fixed biases were observed. Therefore, the data obtained by OpenPose can be improved by offsetting. When measuring the hip flexion-extension ROM, OpenPose can be used. In contrast, when measuring the hip flexion or extension angles only, conventional marker-based systems may be appropriate.

In previous studies involving the measurement of the knee flexion and extension angles during walking or running using Kinect and VICON, the Pearson correlation coefficient between the measurements, r, ranged from 0.43 to 0.69 for walking at $4.8 \mathrm{~km} / \mathrm{h}$ and running at $8.8 \mathrm{~km} / \mathrm{h}[10]$. Similarly, our study revealed that the data obtained by OpenPose and VICON during walking at $4.0 \mathrm{~km} / \mathrm{h}$ and running at $8.5 \mathrm{~km} / \mathrm{h}$ were significantly associated, and $\mathrm{R}^{2}$, which was the coefficient of determination, ranged from 0.52 to 0.92 . In another previous study using Kinect, $r$, which was the Pearson correlation coefficient for the hip flexion-extension ROM during striding, was verified by VICON and measured at 0.49 for walking at $4.5 \mathrm{~km} / \mathrm{h}$ and 0.17 for walking at $6.0 \mathrm{~km} / \mathrm{h}$ [9]. Our results revealed that $\mathrm{R}^{2}$ which was the coefficient of determination between the data obtained by OpenPose and VICON was 0.55 for walking at $4.0 \mathrm{~km} / \mathrm{h}$ and 0.66 for walking at $5.5 \mathrm{~km} / \mathrm{h}$. The Pearson correlation coefficient between the data obtained by Kinect and VICON, r, for the ankle ROM during striding was 0.11 for walking at $4.5 \mathrm{~km} / \mathrm{h}$ and -0.26 for walking at $6.0 \mathrm{~km} / \mathrm{h}$ [9]. On the other hand, the data obtained by OpenPose and VICON showed $\mathrm{R}^{2}$, which was the coefficient of determination, was 0.64 for walking at $4.0 \mathrm{~km} / \mathrm{h}$ and 0.66 for walking at $5.5 \mathrm{~km} / \mathrm{h}$. Currently, OpenPose could be recommended as an easy and economical method for measuring segment and joint angles during walking and running. OpenPose can measure the lower limb angles in the sagittal plane from the images captured using one digital camera, without requiring special equipment or specialists. 
Several limitations of this study should be noted. First, due to the features of OpenPose, it is necessary to correct the data for fixed and proportional biases, and investigate the error between the true values indicating actual joint motion and OpenPose values. Next, the applicability of OpenPose for analyzing faster 3D motion tasks such as jumping and throwing has not been verified yet. We must determine whether OpenPose can be applied for measuring segment and joint angles during faster 3D motion tasks. Furthermore, the participants were limited to young healthy people and did not include patients and the elderly with disorders. Thus, to apply OpenPose for gait analysis during abnormal walking, it is necessary to verify its validity for gait analyses in patients and the elderly.

\section{Conclusions}

This study verified the validity of OpenPose-based gait analysis. The knee and ankle joint angles measured using OpenPose were significantly associated with those measured using VICON, and only fixed biases but no proportional biases were observed. Moreover, most parameters of the lower limb ROM in the sagittal plane had large coefficients of determination, without proportional biases, and the ICCs were moderate to excellent. On the other hand, for most parameters of the lower limb angles in the frontal plane, there were nonsignificant coefficients of determination and poor ICCs $(2,1)$, with fixed and proportional biases. Similarly, the measurements of the hip joint angles in the sagittal plane had nonsignificant coefficients of determination and poor ICCs $(2,1)$, with fixed and proportional biases. Thus, we could measure the lower limb ROM in the sagittal plane using OpenPose from the images captured with one digital camera. Although it is not an alternative to a complete 3D motion analysis system, OpenPose can still be used for gait analysis. OpenPose can reduce analysis costs and times because it is a markerless system without special cameras. 


\section{References}

1. S. Lord, T. Howe, J. Greenland, L. Simpson, L. Rochester, Gait variability in older adults: a structured review of testing protocol and clinimetric properties, Gait Posture 34 (2011) 443-450. https://doi:

10.1016/j.gaitpost.2011.07.010.

2. D.M. Peters, S.L. Fritz, D.E. Krotish, Assessing the Reliability and Validity of a Shorter Walk Test Compared With the 10-Meter Walk Test for Measurements of Gait Speed in Healthy, Older Adults, J Geriatr Phys Ther. 36 (2013) 24-30. https://doi10.1519/JPT.0b013e318248e20d.

3. R.O. Crapo, R. Casaburi, A.L. Coates, P.L. Enright, N.R. MacIntyre, R.T. McKay, D. Johnson, J.S. Wanger, R.J. Zeballos, V. Bittner, C. Mottram, ATS statement: guidelines for the six-minute walk test, Am. J. Respir. Crit. Care Med. 166 (2002), 111-7. http://10.1164/rccm.166/1/111.

4. S. J. Singh, M. D. L. Morgan, S. Scott, Walters D., A. E. Hardman, Development of a shuttle walking test of disability in patients with chronic airways obstruction, Thorax 47 (1992), 1019-24. https://doi.org/10.1136/thx.47.12.1019.

5. D.E. Krebs, J.E. Edelstein, S. Fishman, Reliability of observational kinematic gait analysis, Phys. Ther. 65 (1985) 1027-1033. https://doi.org/10.1093/ptj/65.7.1027.

6. H. Tateuchi, Y. Koyama, H. Akiyama, K. Goto, K. So, Y. Kuroda, N. Ichihashi, Radiographic and clinical factors associated with one-leg standing and gait in patients with mild-to-moderate secondary hip osteoarthritis, Gait Posture 49 (2016) 207-212. https://doi.org/10.1016/j.gaitpost.2016.07.018.

7. Y. Fukumoto, H. Tateuchi, R. Tsukagoshi, Y. Okita, H. Akiyama, K. So, Y. Kuroda, N. Ichihashi N, Effects of highand low-velocity resistance training on gait kinematics and kinetics in individuals with hip osteoarthritis: a randomized controlled trial, Am. J. Phys. Med. Rehab. 96 (2017) 417-423. https://doi:

\subsection{7/PHM.0000000000000640.}

8. R. Baker, Gait analysis methods in rehabilitation, J. Neuroeng. Rehab. 3 (2006) 1-10. https://doi.org/10.1186/17430003-3-4.

9. B.F. Mentiplay, L.G. Perraton, K.J. Bower, Y.H. Pua, R. McGaw, S. Heywood, R. A Clark, Gait assessment using the Microsoft Xbox One Kinect: Concurrent validity and inter-day reliability of spatiotemporal and kinematic variables, 
J. Biomech. 48 (2015) 2166-2170. https://doi.org/10.1016/j.jbiomech.2015.05.021.

10. A. Pfister, A.M. West, S. Bronner, J.A. Noah, Comparative abilities of Microsoft Kinect and Vicon 3D motion capture for gait analysis, J. Med. Eng. Technol. 38 (2014) 272-280. https://doi.org/10.3109/03091902.2014.909540.

11. R.A. Clark, K.J. Bower, B.F. Mentiplay, K. Paterson, Y.H. Pua, Concurrent validity of the Microsoft Kinect for assessment of spatiotemporal gait variables, J Biomech. 46 (2013) 2722-2725.

https://doi.org/10.1016/j.jbiomech.2013.08.011.

12. X. Xu, R.W. McGorry, L.S. Chou, J.H. Lin, C.C. Chang, Accuracy of the Microsoft Kinect for measuring gait parameters during treadmill walking, Gait Posture. 42 (2015) 145-151. https://doi.org/10.1016/j.gaitpost.2015.05.002.

13. B. Galna, G. Barry, D. Jackson, D. Mhiripiri, P. Olivier, L. Rochester, Accuracy of the Microsoft Kinect sensor for measuring movement in people with Parkinson's disease, Gait Posture. 39 (2014) 1062-1068. https://doi.org/10.1016/j.gaitpost.2014.01.008.

14. S. Springer, G.Y. Seligmann, Validity of the Kinect for gait assessment: A focused review, Sensors (Basel) 16 (2016) 194. https://doi.org/10.3390/s16020194.

15. Z. Cao, T. Simon, S.E. Wei, Y. Sheikh. Realtime Multi-Person 2D Pose Estimation using Part Affinity Fields. Computer Vision and Pattern Recognition (2017) https://arxiv.org/abs/1611.08050.

16. Z. Cao, T. Simon, S.E. Wei, Y. Sheikh. OpenPose: Realtime Multi-Person 2D Pose Estimation using Part Affinity Fields. IEEE Transactions on Pattern Analysis and Machine Intelligence (2019) https://arxiv.org/abs/1812.08008.

17. OpenCV 4.1.1, https://opencv.org/ (accessed 18 October 2019).

18. G. Bradski, The OpenCV Library, Dr. Dobb's Journal of Software Tools. (2000). http://www.drdobbs.com/opensource/the-opencv-library/184404319.

19. G. Hidalgo, Z. Cao, T. Simon, S.E. Wei, H. Joo, Y. Sheikh, OpenPose library. https://github.com/CMU-PerceptualComputing-Lab/openpose.

20. M. Ota, H. Tateuchi, T. Hashiguchi, T. Kato, Y. Ogino, N. Ichihashi, Verification of reliability and validity of motion analysis systems during bilateral squat using human pose tracking algorithm, Gait Posture. 80 (2020) 62-67. https://doi.org/10.1016/j.gaitpost.2020.05.027.

21. N. Nakao, T. Sakura, T. Mano, K. Ueda, L. Omura, A. Kimura, Y. Iino, S. Fukashiro, S. Yoshida, Evaluation of 3D 
markerless motion capture accuracy using OpenPose with multiple video cameras. Front. Sports Act. Living. 2 (2020) 50. https://doi.org/10.3389/fspor.2020.00050.

22. M. Zago, M. Luzzago, T. Marangoni, M. De Cecco, M. Tarabini, M.Galli, 3D Tracking of human motion using visual skeletonization and stereoscopic vision. Front. Bioeng. Biotechnol. 8 (2020) 181.

https://doi.org/10.3389/fbioe.2020.00181.

23. D. Winter, Biomechanics and motor control of human movement. John Wiley \& Sons, Inc, Hoboken, 1990.

24. J. Cohen, Statistical Power Analysis for the Behavioral Sciences, second ed., Phycology Press, New York, 1988.

25. T.K. Koo, M.Y. Koch, A Guideline of Selecting and Reporting Intraclass Correlation Coefficients for Reliability Research. J Chiropr Med. 15 (2016) 155-163. https://doi:10.1016/j.jcm.2016.02.012.

26. K. Sato, Y. Nagashima, T. Mano, A. Iwata, T. Toda, Quantifying normal and parkinsonian gait features from home movies: Practical application of a deep learning-based 2D pose estimator. PLoS One. 14 (2019) e0223549. https://doi:10.1371/journal.pone.0223549. 


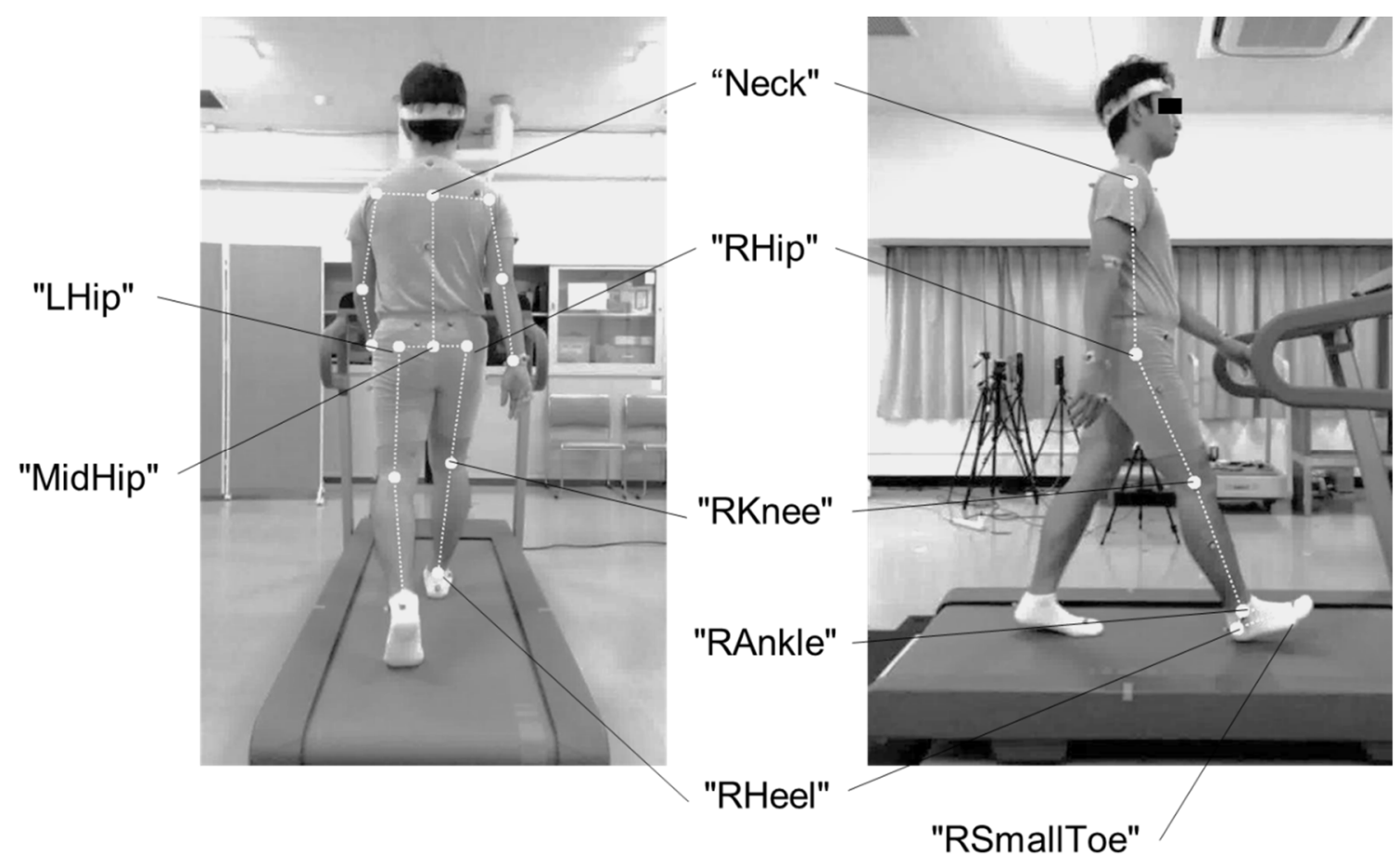
a. The frontal image
b. The sagittal image

Figure 1. Placement and definition of each feature point by OpenPose

The figure shows the feature points estimated by OpenPose in the frontal image (left panel: a) and sagittal image (right panel: b).

Abbreviations: Neck; border between cervical and thoracic vertebrae; RHip; right hip joint, LHip; left hip joint, MidHip; center of RHip and LHip, RKnee; right knee joint, RAnkle; right ankle joint, RHeel; right heel, RSmallToe; right 5th metatarsophalangeal joint.

OpenPose estimates all the feature points from each image. 

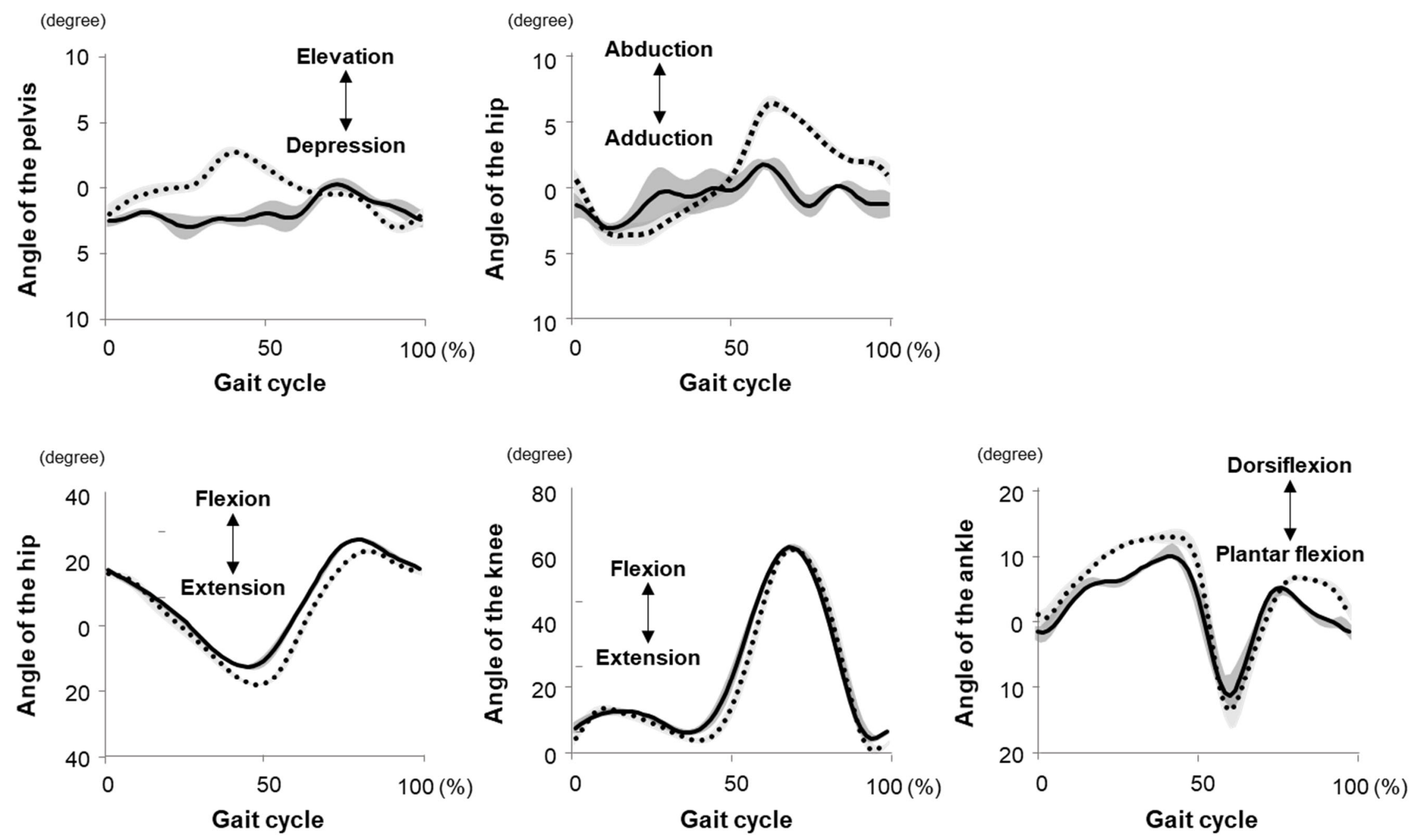

Figure 2. Typical example of temporal changes during moderate walking.

Abbreviations: elevation; pelvic tilt to upper right, depression; pelvic tilt to lower right.

Mean values and standard deviations of four gait cycles in a participant were are shown as lines with bands. Black lines with dark gray bands denote the data obtained by OpenPose, 
and black dotted lines with light gray bands denote the data obtained by VICON. 
Table 1. Definitions of segments and joints based on OpenPose

\begin{tabular}{cl} 
Segments and joints & Angles \\
\hline $\begin{array}{c}\text { Pelvis } \\
\text { Elevation / Depression } \\
\text { Hip }\end{array}$ & The angle of a straight line connecting "RHip" and "LHip" relative to horizontal \\
Flexion / Extension & $\begin{array}{l}\text { The angle of a straight line connecting "RHip" and "RKnee" relative to } \\
\text { a straight line connecting "Neck" and "MidHip" }\end{array}$ \\
Hip & $\begin{array}{l}\text { The angle of the straight line connecting "RHip" and "RKnee" relative to the } \\
\text { perpendicular line connecting "RHip" and "LHip" }\end{array}$ \\
Abduction / Adduction & The angle of "RHip", "RKnee" and "RAnkle" \\
Knee & The angle of a straight line connecting "RHeel" and "RSmallToe" relative to \\
Flexion / Extension & a straight line connecting "RKnee" and "RAnkle" \\
Ankle &
\end{tabular}

Abbreviations : Neck; border between cervical and thoracic vertebrae; RHip; right hip joint, LHip; left hip joint, MidHip;

center of RHip and LHip, RKnee; right knee joint, RAnkle; right ankle joint, RHeel; right heel, RSmallToe; right 5th metatarsophalangeal joint. 
Table 2. Mean values \pm standard deviation (degree) for each peak angle and range measured using OpenPose and VICON.

\begin{tabular}{|c|c|c|c|c|c|c|c|c|c|}
\hline \multirow{2}{*}{ Motion } & \multirow{2}{*}{$\begin{array}{r}\text { Velocity } \\
\text { Device }\end{array}$} & \multicolumn{2}{|c|}{ Slow walking } & \multicolumn{2}{|c|}{ Moderate walking } & \multicolumn{2}{|c|}{ Fast walking } & \multicolumn{2}{|c|}{ Running } \\
\hline & & OpenPose & VICON & OpenPose & VICON & OpenPose & VICON & OpenPose & VICON \\
\hline \multicolumn{10}{|l|}{ a. Pelvis } \\
\hline Elevation & & $2.04 \pm 1.30$ & $3.19 \pm 1.54$ & $1.49 \pm 1.03$ & $4.56 \pm 1.92$ & $1.45 \pm 0.91$ & $5.57 \pm 1.77$ & $2.23 \pm 1.08$ & $3.87 \pm 1.52$ \\
\hline Depression & & $3.24 \pm 0.83$ & $4.28 \pm 1.48$ & $2.48 \pm 0.79$ & $5.30 \pm 1.78$ & $2.50 \pm 1.04$ & $6.30 \pm 1.52$ & $2.54 \pm 1.41$ & $4.25 \pm 1.31$ \\
\hline Range & & $5.28 \pm 1.61$ & $7.47 \pm 2.27$ & $3.89 \pm 1.07$ & $9.86 \pm 3.04$ & $4.40 \pm 1.09$ & $11.87 \pm 2.58$ & $4.77 \pm 1.59$ & $8.12 \pm 1.90$ \\
\hline \multicolumn{10}{|l|}{ b. Hip } \\
\hline Flexion & & $23.29 \pm 3.35$ & $26.36 \pm 4.61$ & $26.59 \pm 2.42$ & $28.23 \pm 4.43$ & $27.75 \pm 2.26$ & $31.52 \pm 4.32$ & $26.86 \pm 4.31$ & $35.33 \pm 5.67$ \\
\hline Extension & & $12.18 \pm 2.84$ & $12.31 \pm 5.61$ & $14.69 \pm 1.71$ & $16.14 \pm 5.24$ & $18.35 \pm 2.47$ & $18.80 \pm 5.03$ & $17.87 \pm 1.61$ & $10.79 \pm 3.76$ \\
\hline Range & & $35.48 \pm 4.30$ & $38.66 \pm 4.54$ & $41.28 \pm 2.95$ & $44.37 \pm 2.80$ & $46.09 \pm 2.86$ & $50.32 \pm 3.45$ & $44.73 \pm 4.59$ & $46.12 \pm 5.20$ \\
\hline Abduction & & $2.24 \pm 1.77$ & $6.20 \pm 2.82$ & $2.17 \pm 1.78$ & $7.46 \pm 2.71$ & $2.14 \pm 1.81$ & $8.50 \pm 2.29$ & $0.48 \pm 1.93$ & $4.89 \pm 2.02$ \\
\hline Adduction & & $5.07 \pm 1.66$ & $4.86 \pm 2.46$ & $5.99 \pm 1.58$ & $6.57 \pm 2.78$ & $6.31 \pm 1.41$ & $7.13 \pm 2.82$ & $5.87 \pm 2.75$ & $9.16 \pm 3.03$ \\
\hline Range & & $7.31 \pm 1.46$ & $11.06 \pm 3.17$ & $8.16 \pm 1.17$ & $14.03 \pm 2.67$ & $8.45 \pm 1.50$ & $15.63 \pm 2.70$ & $6.06 \pm 2.01$ & $14.05 \pm 3.32$ \\
\hline \multicolumn{10}{|l|}{ c. Knee } \\
\hline Flexion & & $58.64 \pm 4.52$ & $55.50 \pm 4.22$ & $63.46 \pm 4.09$ & $60.93 \pm 3.66$ & $63.23 \pm 4.07$ & $61.46 \pm 3.39$ & $81.64 \pm 8.18$ & $79.91 \pm 9.21$ \\
\hline Extension & & $-2.39 \pm 4.42$ & $2.06 \pm 4.05$ & $-5.00 \pm 3.34$ & $1.38 \pm 3.63$ & $-7.67 \pm 3.55$ & $-0.81 \pm 3.87$ & $-14.65 \pm 4.03$ & $-6.57 \pm 3.91$ \\
\hline Range & & $56.25 \pm 5.42$ & $57.57 \pm 5.26$ & $58.46 \pm 4.59$ & $62.31 \pm 4.45$ & $55.56 \pm 4.61$ & $60.65 \pm 4.52$ & $66.99 \pm 6.44$ & $73.34 \pm 7.62$ \\
\hline \multicolumn{10}{|l|}{ d. Ankle } \\
\hline Dorsiflexion & & $11.68 \pm 2.88$ & $15.26 \pm 2.94$ & $11.57 \pm 3.53$ & $15.05 \pm 3.93$ & $8.98 \pm 3.53$ & $12.10 \pm 3.24$ & $17.76 \pm 2.41$ & $24.64 \pm 2.98$ \\
\hline Plantar flexion & & $7.72 \pm 5.69$ & $8.94 \pm 6.08$ & $14.09 \pm 6.14$ & $17.23 \pm 7.96$ & $18.46 \pm 7.35$ & $21.88 \pm 7.57$ & $22.78 \pm 8.06$ & $25.46 \pm 10.22$ \\
\hline Range & & $19.40 \pm 6.25$ & $24.20 \pm 5.49$ & $25.67 \pm 6.39$ & $32.27 \pm 7.80$ & $27.43 \pm 6.77$ & $33.98 \pm 7.19$ & $40.54 \pm 8.24$ & $50.10 \pm 9.45$ \\
\hline
\end{tabular}

Abbreviations: elevation; pelvic tilt to upper right, depression; pelvic tilt to lower right.

Negative values for knee extension angles indicate flexion positions. 
Table 3. The regression models and the intraclass correlation coefficients $[\mathrm{ICC}(2,1)]$ for each peak angle and range measured using OpenPose and VICON.

a. Pelvis

\begin{tabular}{rrrrrrrr}
\hline Motion & Velocity & Unstandardized coefficients B & Constant & $95 \%$ CI for B (p value) & $\mathrm{R}^{2}$ & ICC $(2,1)$ & $95 \%$ CI for ICC $(2,1)$ \\
\hline \multirow{5}{*}{ Elevation } & Slow walking & 0.107 & 3.029 & $-0.430-0.645(\mathrm{p}=0.682)$ & 0.008 & 0.068 & $-0.220-0.401$ \\
& Moderate walking & -0.092 & 4.701 & $-0.920-0.736(\mathrm{p}=0.820)$ & 0.002 & -0.014 & $-0.122-0.171$ \\
& Fast walking & 0.127 & 5.381 & $-0.730-0.984(\mathrm{p}=0.761)$ & 0.004 & 0.010 & $-0.050-0.126$ \\
& Running & $\mathbf{0 . 6 0 2}$ & $\mathbf{2 . 5 2 8}$ & $\mathbf{0 . 0 3 8}-\mathbf{1 . 1 6 8}(\mathbf{p}=\mathbf{0 . 0 3 8})$ & $\mathbf{0 . 1 8 1}$ & 0.229 & $-0.103-0.554$ \\
& Slow walking & -0.122 & -4.610 & $-0.930-0.687(\mathrm{p}=0.758)$ & 0.005 & -0.045 & $-0.330-0.306$ \\
& Moderate walking & 0.633 & -3.727 & $-0.319-1.585(\mathrm{p}=0.182)$ & 0.080 & 0.069 & $-0.077-0.293$ \\
& Fast walking & $\mathbf{0 . 6 4 7}$ & $\mathbf{- 4 . 6 8 8}$ & $\mathbf{0 . 0 6 6}-\mathbf{1 . 2 2 8}(\mathbf{p}=\mathbf{0 . 0 3 1})$ & $\mathbf{0 . 1 9 5}$ & 0.078 & $-0.048-0.311$ \\
& Running & 0.077 & -4.051 & $-0.332-0.487(\mathrm{p}=0.699)$ & 0.007 & 0.047 & $-0.154-0.322$ \\
& Slow walking & -0.370 & 9.416 & $-1.001-0.261(\mathrm{p}=0.236)$ & 0.066 & 0.156 & $-0.407-0.194$ \\
& Moderate walking & -1.041 & 13.992 & $-2.186-0.104(\mathrm{p}=0.073)$ & 0.139 & -0.055 & $-0.149-0.145$ \\
& Fast walking & 0.264 & 10.827 & $-0.769-1.296(\mathrm{p}=0.602)$ & 0.013 & 0.009 & $-0.028-0.084$ \\
& Running & 0.185 & 7.234 & $-0.337-0.708(\mathrm{p}=0.470)$ & 0.024 & 0.055 & $-0.084-0.271$ \\
\hline
\end{tabular}




\begin{tabular}{|c|c|c|c|c|c|c|c|}
\hline Motion & Velocity & Unstandardized coefficients B & Constant & $95 \% \mathrm{CI}$ for B ( $\mathrm{p}$ value) & $\mathrm{R}^{2}$ & $\operatorname{ICC}(2,1)$ & $95 \%$ CI for $\operatorname{ICC}(2,1)$ \\
\hline \multirow{4}{*}{ Flexion } & Slow walking & 0.547 & 13.621 & $-0.011-1.105(\mathrm{p}=0.054)$ & 0.158 & 0.299 & $-0.057-0.607$ \\
\hline & Moderate walking & 0.366 & 18.507 & $-0.428-1.159(p=0.349)$ & 0.040 & 0.157 & $-0.216-0.506$ \\
\hline & Fast walking & 0.111 & 28.442 & $-0.732-0.954(p=0.787)$ & 0.003 & 0.031 & $-0.195-0.325$ \\
\hline & Running & 0.650 & 17.874 & $0.145-1.155(p=0.014)$ & 0.244 & 0.199 & $-0.097-0.536$ \\
\hline \multirow{4}{*}{ Extension } & Slow walking & 1.146 & 1.655 & $0.436-1.856(p=0.003)$ & $\underline{0.338}$ & 0.479 & $0.093-0.737$ \\
\hline & Moderate walking & 1.137 & 0.557 & $-0.120-2.393(p=0.074)$ & 0.138 & 0.211 & $-0.178-0.553$ \\
\hline & Fast walking & 0.699 & -5.966 & $-0.145-1.544(\mathrm{p}=0.100)$ & 0.118 & 0.279 & $-0.143-0.611$ \\
\hline & Running & 0.203 & -7.157 & $-0.827-1.234(p=0.686)$ & 0.008 & 0.016 & $-0.066-0.164$ \\
\hline \multirow{4}{*}{ Range } & Slow walking & 0.868 & 7.886 & $0.603-1.132(p<0.001)$ & $\underline{0.678}$ & $\underline{0.656}$ & $-0.031-0.882$ \\
\hline & Moderate walking & 0.704 & 15.290 & $0.423-0.986(p<0.001)$ & $\underline{0.550}$ & 0.473 & $-0.104-0.796$ \\
\hline & Fast walking & 0.985 & 4.924 & $0.675-1.294(p<0.001)$ & $\underline{0.664}$ & 0.426 & $-0.082-0.784$ \\
\hline & Running & 1.022 & 0.411 & $0.806-1.238(p<0.001)$ & $\underline{0.813}$ & $\underline{0.864}$ & $0.625-0.946$ \\
\hline \multirow{4}{*}{ Abduction } & Slow walking & 1.085 & 3.768 & $0.567-1.602(p<0.001)$ & $\underline{0.462}$ & 0.255 & $-0.090-0.622$ \\
\hline & Moderate walking & 1.111 & 5.042 & $0.651-1.571(p<0.001)$ & $\underline{0.533}$ & 0.184 & $-0.052-0.541$ \\
\hline & Fast walking & 0.779 & 6.837 & $0.339-1.219(p=0.001)$ & $\underline{0.380}$ & 0.105 & $-0.036-0.387$ \\
\hline & Running & 0.119 & 5.018 & $-0.383-0.622(\mathrm{p}=0.622)$ & 0.013 & 0.031 & $-0.070-0.215$ \\
\hline \multirow{4}{*}{ Adduction } & Slow walking & 0.890 & -0.352 & $0.365-1.415(p=0.002)$ & $\underline{0.359}$ & $\underline{0.563}$ & $0.212-0.785$ \\
\hline & Moderate walking & 0.979 & -0.710 & $0.332-1.626(p=0.005)$ & $\underline{0.309}$ & 0.472 & $0.105-0.729$ \\
\hline & Fast walking & 0.922 & -1.306 & $0.135-1.709(p=0.024)$ & 0.211 & 0.353 & $-0.026-0.651$ \\
\hline & Running & 0.585 & -5.527 & $0.124-1.046(p=0.016)$ & $\underline{0.270}$ & 0.337 & $-0.094-0.674$ \\
\hline \multirow{4}{*}{ Range } & Slow walking & 0.649 & 6.319 & $-0.267-1.565(p=0.156)$ & 0.089 & 0.107 & $-0.102-0.381$ \\
\hline & Moderate walking & 0.645 & 8.762 & $-0.319-1.609(p=0.179)$ & 0.080 & 0.042 & $-0.049-0.205$ \\
\hline & Fast walking & 0.734 & 9.428 & $0.006-1.462(p=0.048)$ & 0.166 & 0.054 & $-0.040-0.238$ \\
\hline & Running & 0.317 & 12.136 & $-0.473-1.107(p=0.413)$ & 0.034 & 0.032 & $-0.050-0.186$ \\
\hline
\end{tabular}




\begin{tabular}{|c|c|c|c|c|c|c|c|}
\hline Motion & Velocity & Unstandardized coefficients B & Constant & 95\% CI for B ( $\mathrm{p}$ value) & $\mathrm{R}^{2}$ & $\operatorname{ICC}(2,1)$ & $95 \% \mathrm{CI}$ for $\operatorname{ICC}(2,1)$ \\
\hline \multirow{4}{*}{ Flexion } & Slow walking & 0.786 & 9.430 & $0.562-1.009(p<0.001)$ & $\underline{0.707}$ & $\underline{0.670}$ & $-0.040-0.891$ \\
\hline & Moderate walking & 0.706 & 16.107 & $0.464-0.949(p<0.001)$ & $\underline{0.623}$ & $\underline{0.652}$ & $0.054-0.869$ \\
\hline & Fast walking & 0.574 & 25.179 & $0.307-0.841(p<0.001)$ & $\underline{0.475}$ & $\underline{0.617}$ & $0.243-0.822$ \\
\hline & Running & 1.079 & -8.174 & $0.935-1.222(p<0.001)$ & $\underline{0.917}$ & $\underline{0.934}$ & $0.791-0.975$ \\
\hline \multirow{4}{*}{ Extension } & Slow walking & 0.720 & -3.784 & $0.469-0.972(p<0.001)$ & $\underline{0.616}$ & $\underline{0.507}$ & $-0.101-0.821$ \\
\hline & Moderate walking & 0.874 & -5.745 & $0.588-1.159(p<0.001)$ & $\underline{0.647}$ & 0.301 & $-0.051-0.694$ \\
\hline & Fast walking & 0.698 & -4.552 & $0.329-1.068(p=0.001)$ & $\underline{0.412}$ & 0.237 & $-0.077-0.607$ \\
\hline & Running & 0.696 & -3.629 & $0.397-0.995(p<0.001)$ & $\underline{0.515}$ & 0.234 & $-0.056-0.614$ \\
\hline \multirow{4}{*}{ Range } & Slow walking & 0.911 & 6.341 & $0.764-1.058(p<0.001)$ & $\underline{0.883}$ & $\underline{0.914}$ & $0.703-0.968$ \\
\hline & Moderate walking & 0.849 & 12.700 & $0.640-1.057(p<0.001)$ & $\underline{0.764}$ & $\underline{0.644}$ & $-0.086-0.892$ \\
\hline & Fast walking & 0.816 & 15.299 & $0.575-1.057(p<0.001)$ & $\underline{0.692}$ & $\underline{0.515}$ & $-0.089-0.835$ \\
\hline & Running & 1.136 & -2.757 & $0.988-1.284(p<0.001)$ & $\underline{0.920}$ & $\underline{0.674}$ & $-0.050-0.915$ \\
\hline
\end{tabular}


d. Ankle

\begin{tabular}{|c|c|c|c|c|c|c|c|}
\hline Motion & Velocity & Unstandardized coefficients B & Constant & $95 \%$ CI for B ( $p$ value) & $\mathrm{R}^{2}$ & $\operatorname{ICC}(2,1)$ & $95 \%$ CI for $\operatorname{ICC}(2,1)$ \\
\hline \multirow{4}{*}{ Dorsiflexion } & Slow walking & 0.376 & 10.863 & $-0.044-0.797(p=0.077)$ & 0.135 & 0.213 & $-0.103-0.532$ \\
\hline & Moderate walking & 0.655 & 7.462 & $0.257-1.054(p=0.003)$ & $\underline{0.346}$ & 0.413 & $-0.065-0.724$ \\
\hline & Fast walking & 0.604 & 6.677 & $0.299-0.909(p<0.001)$ & $\underline{0.433}$ & 0.465 & $-0.071-0.769$ \\
\hline & Running & 0.566 & 14.580 & $0.079-1.054(p=0.025)$ & 0.209 & 0.107 & $-0.059-0.382$ \\
\hline \multirow{4}{*}{ Plantar flexion } & Slow walking & 0.506 & -5.031 & $0.089-0.922(p=0.020)$ & 0.224 & 0.472 & $0.099-0.731$ \\
\hline & Moderate walking & 0.875 & -4.889 & $0.452-1.298(\mathrm{p}<0.001)$ & $\underline{0.456}$ & $\underline{0.603}$ & $0.249-0.810$ \\
\hline & Fast walking & 0.697 & -9.020 & $0.362-1.031(\mathrm{p}<0.001)$ & $\underline{0.459}$ & $\underline{0.620}$ & $0.255-0.822$ \\
\hline & Running & 0.910 & -4.730 & $0.519-1.301(p<0.001)$ & $\underline{0.515}$ & $\underline{0.677}$ & $0.387-0.846$ \\
\hline \multirow{4}{*}{ Range } & Slow walking & 0.613 & 12.308 & $0.334-0.891(\mathrm{p}<0.001)$ & $\underline{0.486}$ & $\underline{0.524}$ & $-0.034-0.802$ \\
\hline & Moderate walking & 0.980 & 7.122 & $0.658-1.302(\mathrm{p}<0.001)$ & $\underline{0.644}$ & $\underline{0.554}$ & $-0.093-0.841$ \\
\hline & Fast walking & 0.865 & 10.258 & $0.592-1.137(\mathrm{p}<0.001)$ & $\underline{0.663}$ & $\underline{0.568}$ & $-0.095-0.852$ \\
\hline & Running & 0.885 & 14.223 & $0.563-1.207(p<0.001)$ & $\underline{0.596}$ & 0.486 & $-0.103-0.808$ \\
\hline
\end{tabular}

Abbreviations: elevation; pelvic tilt to upper right, depression; pelvic tilt to lower right, CI, confidence intervals; ICC, intraclass correlation coefficient.

The linear regression analyses were performed by using the data obtained by OpenPose as independent variable and the data obtained by VICON as dependent variable.

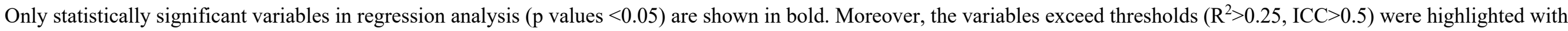
underlined. 
Table 4. Fixed and proportional biases by Bland-Altman analysis for each peak angle and range measured using OpenPose and VICON.

a. Pelvis

\begin{tabular}{|c|c|c|c|c|c|c|}
\hline \multirow{2}{*}{ Motion } & \multirow{2}{*}{\multicolumn{2}{|c|}{ Velocity }} & \multicolumn{3}{|c|}{ Fixed bias } & \multirow{2}{*}{$\frac{\text { Proportional bias }}{\text { Regression equation ( } \mathrm{p} \text { value) }}$} \\
\hline & & & Bias & 95\% CI for bias ( $\mathrm{p}$ value) & Upper LoA-Lower LoA & \\
\hline \multirow{4}{*}{ Elevation } & Slow & walking & -1.234 & $-2.034--0.430(p=0.004)$ & $-4.947-2.479$ & $y=-0.484-0.287 x(p=0.459)$ \\
\hline & Moderate & walking & -3.075 & $-4.014--2.136(p<0.001)$ & $-7.432-1.283$ & $y=0.442-1.162 x(p=0.005)$ \\
\hline & Fast & walking & -4.111 & $-4.928--3.294(p<0.001)$ & $-7.902--0.321$ & $y=-0.240-1.103 x(p=0.003)$ \\
\hline & & Running & -1.641 & $-2.251--1.031(p<0.001)$ & $-4.472-1.191$ & $y=-0.185-0.477 x(p=0.080)$ \\
\hline \multirow{4}{*}{ Depression } & Slow & walking & 1.183 & $0.351-2.015(p=0.007)$ & $-2.678-5.044$ & $y=-4.064-1.375 x(p=0.001)$ \\
\hline & Moderate & walking & 2.817 & $2.085-3.548(p<0.001)$ & $-0.579-6.212$ & $y=-1.481-1.105 x(p<0.001)$ \\
\hline & Fast & walking & 3.807 & $3.210-4.403(p<0.001)$ & $1.037-6.576$ & $y=1.534-0.516 x \quad(p=0.053)$ \\
\hline & & Running & 1.711 & $0.931-2.490(p<0.001)$ & $-1.907-5.328$ & $y=2.167+0.135 x \quad(p=0.734)$ \\
\hline \multirow{4}{*}{ Range } & Slow & walking & -2.417 & $-3.801--1.033(p=0.001)$ & $-8.842-4.008$ & $y=4.470-1.072 x \quad(p=0.051)$ \\
\hline & Moderate & walking & -5.891 & $-7.408--4.374(p<0.001)$ & $-12.932-1.150$ & $y=8.107-2.024 x(p<0.001)$ \\
\hline & Fast & walking & -7.918 & $-9.052--6.784(p<0.001)$ & $-13.183--2.653$ & $y=2.232-1.283 x(p<0.001)$ \\
\hline & & Running & -3.351 & $-4.315--2.387(p<0.001)$ & $-7.826-1.124$ & $y=-1.366-0.308 x(p=0.401)$ \\
\hline
\end{tabular}




\begin{tabular}{|c|c|c|c|c|c|c|}
\hline \multirow{2}{*}{ Motion } & \multirow{2}{*}{\multicolumn{2}{|c|}{ Velocity }} & \multicolumn{3}{|c|}{ Fixed bias } & \multirow{2}{*}{$\begin{array}{c}\text { Proportional bias } \\
\text { Regression equation ( } \mathrm{p} \text { value })\end{array}$} \\
\hline & & & Bias & $95 \%$ CI for bias ( $p$ value) & LoA (Upper-Lower) & \\
\hline \multirow{4}{*}{ Flexion } & Slow & walking & -3.065 & $-4.962--1.168(p=0.003)$ & $-11.869-5.740$ & $y=8.049-0.448 x(p=0.112)$ \\
\hline & Moderate & walking & -1.642 & $-3.586-0.302(p=0.094)$ & $-10.666-7.381$ & $y=23.723-0.926 x(p=0.006)$ \\
\hline & Fast & walking & -3.775 & $-5.782--1.767(p=0.001)$ & $-13.092-5.543$ & $y=28.425-1.087 x(p=0.004)$ \\
\hline & & Running & -8.473 & $-10.650--6.297(p<0.001)$ & $-18.576-1.630$ & $y=2.776-0.362 x(p=0.149)$ \\
\hline \multirow{4}{*}{ Extension } & Slow & walking & 0.124 & $-1.813-2.060(p=0.896)$ & $-8.864-9.112$ & $y=-9.73-0.805 x(p<0.001)$ \\
\hline & Moderate & walking & 1.449 & $-0.606-3.505(\mathrm{p}=0.158)$ & $-8.090-10.989$ & $y=-18.963-1.324 x(p<0.001)$ \\
\hline & Fast & walking & 0.452 & $-1.567-2.471(p=0.648)$ & $-8.921-9.825$ & $y=-17.381-0.960 x(p=0.001)$ \\
\hline & & Running & -7.079 & $-8.751--5.408(p<0.001)$ & $-14.838-0.679$ & $y=-25.712-1.300 x(p<0.001)$ \\
\hline \multirow{4}{*}{ Range } & Slow & walking & -3.189 & $-4.303--2.075(p<0.001)$ & $-8.359-1.982$ & $y=-1.054-0.058 x(p=0.669)$ \\
\hline & Moderate & walking & -3.092 & $-3.966--2.218(p<0.001)$ & $-7.149-0.966$ & $y=-5.635+0.059 x(p=0.721)$ \\
\hline & Fast & walking & -4.227 & $-5.072--3.382(p<0.001)$ & $-8.147--0.307$ & $y=5.782-0.208 x(p=0.138)$ \\
\hline & & Running & -1.394 & $-2.344--0.444(p=0.006)$ & $-5.803-3.016$ & $y=4.566-0.131 x(p=0.187)$ \\
\hline \multirow{4}{*}{ Abduction } & Slow & walking & -3.957 & $-4.833--3.081(p<0.001)$ & $-8.025-0.111$ & $y=-1.676-0.541 \times(p=0.005)$ \\
\hline & Moderate & walking & -5.283 & $-6.068--4.498(p<0.001)$ & $-8.927--1.638$ & $y=-2.996-0.475 x(p=0.007)$ \\
\hline & Fast & walking & -6.366 & $-7.147--5.583(p<0.001)$ & $-9.994--2.736$ & $y=-4.832-0.288 x(p=0.172)$ \\
\hline & & Running & -4.470 & $-5.540--3.399(p<0.001)$ & $-9.439-0.499$ & $y=-4.052-0.145 x \quad(p=0.692)$ \\
\hline \multirow{4}{*}{ Adduction } & Slow & walking & -0.205 & $-1.041-0.630(p=0.616)$ & $-4.082-3.672$ & $y=-2.604-0.483 \times(p=0.027)$ \\
\hline & Moderate & walking & 0.584 & $-0.390-1.559(p=0.227)$ & $-3.94-5.108$ & $y=-3.769-0.693 \times(p=0.003)$ \\
\hline & Fast & walking & 0.812 & $-0.247-1.871(p=0.126)$ & $-4.103-5.727$ & $y=-5.101-0.880 \times(p=0.001)$ \\
\hline & & Running & 4.022 & $2.435-5.610(p<0.001)$ & $-3.348-11.393$ & $y=-0.338-0.559 x(p=0.051)$ \\
\hline \multirow{4}{*}{ Range } & Slow & walking & -3.752 & $-5.049-2.455(p<0.001)$ & $-9.771-2.268$ & $y=5.975-1.059 x(p<0.001)$ \\
\hline & Moderate & walking & -5.867 & $-6.961--4.773(p<0.001)$ & $-10.944--0.790$ & $y=6.553-1.118 x(p<0.001)$ \\
\hline & Fast & walking & -7.177 & $-8.231--6.124(p<0.001)$ & $-12.067--2.288$ & $y=2.298-0.787 \times(p=0.004)$ \\
\hline & & Running & -8.492 & $-10.139--6.845(p<0.001)$ & $-16.137--0.847$ & $y=1.953-1.006 x(p=0.002)$ \\
\hline
\end{tabular}




\begin{tabular}{|c|c|c|c|c|c|c|}
\hline \multirow{2}{*}{ Motion } & & \multirow{2}{*}{ Velocity } & \multicolumn{3}{|c|}{ Fixed bias } & \multirow{2}{*}{$\frac{\text { Proportional bias }}{\text { Regression equation ( } \mathrm{p} \text { value) }}$} \\
\hline & & & Bias & $95 \%$ CI for bias ( $\mathrm{p}$ value) & LoA (Upper-Lower) & \\
\hline \multirow{4}{*}{ Flexion } & Slow & walking & 3.140 & $2.093-4.188(p<0.001)$ & $-1.722-8.003$ & $y=-1.065+0.074 x(p=0.562)$ \\
\hline & Moderate & walking & 2.529 & $1.455-3.604(p<0.001)$ & $-2.459-7.519$ & $y=-5.202+0.124 x(p=0.403)$ \\
\hline & Fast & walking & 1.770 & $0.501-3.039(p=0.008)$ & $-4.119-7.659$ & $y=-11.680+0.216 x(p=0.246)$ \\
\hline & & Running & 1.736 & $0.583-2.890(p=0.005)$ & $-3.618-7.090$ & $y=-11.564-0.122 x(p=0.065)$ \\
\hline \multirow{4}{*}{ Extension } & Slow & walking & 4.452 & $3.269-5.635(p<0.001)$ & $-1.039-9.942$ & $\mathrm{y}=4.436+0.096 \mathrm{x}(\mathrm{p}=0.522)$ \\
\hline & Moderate & walking & 6.376 & $5.449-7.304(p<0.001)$ & $2.071-10.681$ & $\mathrm{y}=6.542-0.091 \mathrm{x}(\mathrm{p}=0.521)$ \\
\hline & Fast & walking & 6.869 & $5.533-8.198(p<0.001)$ & $0.681-13.050$ & $y=7.304-0.103 x(p=0.608)$ \\
\hline & & Running & 8.084 & $6.824-9.344(p<0.001)$ & $2.234-13.934$ & $y=7.707+0.036 x(p=0.839)$ \\
\hline \multirow{4}{*}{ Range } & Slow & walking & -1.312 & $-2.100--0.524(p=0.002)$ & $-4.969-2.345$ & $y=-3.140+0.032 x(p=0.674)$ \\
\hline & Moderate & walking & -3.846 & $-4.806--2.886(p<0.001)$ & $-8.303-0.610$ & $y=-5.739+0.031 x(p=0.779)$ \\
\hline & Fast & walking & -5.100 & $-6.216--3.976(p<0.001)$ & $-10.293-0.101$ & $y=-6.269+0.020 x(p=0.877)$ \\
\hline & & Running & -6.348 & $-7.329--5.367(p<0.001)$ & $-10.901--1.795$ & $y=5.722-0.127 x(p=0.010)$ \\
\hline
\end{tabular}




\begin{tabular}{|c|c|c|c|c|c|c|}
\hline \multirow{2}{*}{ Motion } & \multirow{2}{*}{\multicolumn{2}{|c|}{ Velocity }} & \multicolumn{3}{|c|}{ Fixed bias } & \multirow{2}{*}{$\begin{array}{c}\text { Proportional bias } \\
\text { Regression equation ( } \mathrm{p} \text { value) }\end{array}$} \\
\hline & & & Bias & $95 \%$ CI for bias ( $\mathrm{p}$ value) & LoA (Upper-Lower) & \\
\hline \multirow{4}{*}{ Dorsiflexion } & Slow & walking & -3.581 & $-4.964--2.199(p<0.001)$ & $-9.998-2.835$ & $y=-3.139-0.033 x(p=0.911)$ \\
\hline & Moderate & walking & -3.472 & $-4.908--2.035(p<0.001)$ & $-10.140-3.196$ & $y=-1.665-0.136 x \quad(p=0.537)$ \\
\hline & Fast & walking & -3.120 & $-4.306--1.934(p<0.001)$ & $-8.626-2.385$ & $y=-4.216+0.104 x(p=0.596)$ \\
\hline & & Running & -6.877 & $-8.081--5.672(p<0.001)$ & $-12.467--1.286$ & $y=-0.694-0.292 x(p=0.267)$ \\
\hline \multirow{4}{*}{ Plantar flexion } & Slow & walking & 1.214 & $-1.341-3.770(p=0.336)$ & $-10.647-13.075$ & $\mathrm{y}=0.460-0.091 \mathrm{x}(\mathrm{p}=0.726)$ \\
\hline & Moderate & walking & 3.132 & $0.631-5.634(p=0.016)$ & $-8.479-14.743$ & $y=-1.680-0.307 x(p=0.109)$ \\
\hline & Fast & walking & 3.423 & $0.890-5.955(p=0.010)$ & $-8.331-15.177$ & $y=2.741-0.034 x(p=0.858)$ \\
\hline & & Running & 2.688 & $-0.336-5.711(\mathrm{p}=0.079)$ & $-11.347-16.722$ & $y=-3.960-0.276 x(p=0.120)$ \\
\hline \multirow{4}{*}{ Range } & Slow & walking & -4.795 & $-6.746-2.845(p<0.001)$ & $-13.849-4.259$ & $y=-8.105+0.125 x(p=0.406)$ \\
\hline & Moderate & walking & -6.604 & $-8.569--4.639(p<0.001)$ & $-15.723-2.515$ & $y=-0.218-0.220 x(p=0.129)$ \\
\hline & Fast & walking & -6.543 & $-8.349--4.737(p<0.001)$ & $-14.927-1.841$ & $y=-4.507-0.066 x(p=0.632)$ \\
\hline & & Running & -9.564 & $-12.131--6.997(p<0.001)$ & $-21.479-2.351$ & $y=-2.578-0.154 x(p=0.322)$ \\
\hline
\end{tabular}

Abbreviations: elevation; pelvic tilt to upper right, depression; pelvic tilt to lower right, CI, confidence interval; LoA, limit of agreement.

In the regression equation, $\mathrm{x}$ denotes mean between the data obtained by OpenPose and VICON, y denotes difference between the data obtained by OpenPose and VICON. Only statistically significant variables ( $\mathrm{p}$ values $<0.05$ ) are shown in bold. 